\title{
The most important echocardiographic characteristics in hypertensive outpatients
}

\author{
Yvonne Vukušić-Janjić ${ }^{1 *}$, Mario Ivanuša ${ }^{2}$ \\ 'Panaceja Polyclinic, Županja, Croatia \\ ${ }^{2}$ Institute for Cardiovascular Prevention and Rehabilitation, Zagreb, Croatia
}

Background: Arterial hypertension is the most important risk factor for premature cardiovascular disease. The prevalence of hypertensive heart disease (HHD) in the population of hypertensive outpatients compared to other cardiac pathologies was clearly overrepresented. ${ }^{1-4}$ The aim of this study was to examine the frequency and severity of the left ventricular hypertrophy $(\mathrm{LVH})$ suggesting the presence of $\mathrm{HHD}$, and to determine the severity of the left atrial enlargement by estimating the volume index as a prognostic marker for adverse cardiovascular outcomes.

Methods: The 3-month, unselected samples of consecutive patients referred to outpatient transthoracic echocardiography (TTE) were included. Conventional TTE were performed according to guidelines using a commercial ultrasound system (GE Logique500PRO). Analysis of demographic data, left ventricular ejection fraction (LVEF), LA enlargement and presence of HHD were performed.

Results: Out of the group of 200 outpatients (123 women, $62 \%$; 77 men, 38\%) we found $156(78 \%)$ patients $(66 \%$ female and $34 \%$ male) suffering from LVH. The average age of patients with LVH was 64.4 years. The average LVEF was $65 \%$, and the value of LVEF below $50 \%$ was found in 10 patients. HHD was strongly suggestive in 95 (48\%) female and $49(25 \%)$ men. Severe LA enlargement was found in $32 \%$, moderate in $18 \%$, and mild in $10 \%$ of all cases.
Conclusions: In the group of outpatients referred to TTE, HHD is a common finding, especially among women. Since there is a wide range of antihypertensive drugs, echocardiography can be used as guidance for the therapy. This is a very important issue for high-risk patients with severe LA enlargement, where the proper therapeutic option can be life saving.

KEYWORDS: echocardiography, hypertension, hypertensive heart disease, left atrial size, echocardiographic therapy guidance

\section{Received: $20^{\text {th }}$ Mar 2013}

*Address for correspondence: Poliklinika Panaceja, Trg prof. Martina Robotića 8, HR-32270 Županja, Croatia.

Phone: +385-32-830094

E-mail: yvonne@janjic.net

\section{Literature}

1. Karabinos I, Grassos C, Kostaki P, Kranidis A. Echocardiography in the evaluation of a hypertensive patient: an invaluable tool or simply following the routine? Hellenic J Cardiol. 2013;54(1):47-57.

2. Patel DA, Lavie CJ, Milani RV, Shah S, Gilliland Y. Clinical Implications of left atrial enlargement: a review. Ochsner J. 2009;9(4):191-6.

3. Di Bello V, Galderisi M, de Gregorio C, Ansalone G, Dini FL, Di Salvo G, et al; Working Group of Echocardiography of the Italian Society of Cardiology (SIC), Italy. New echocardiographic technologies in the clinical management of hypertensive heart disease. J Cardiovasc Med (Hagerstown). 2007;8(12):997-1006.

4. Suárez C, Villar J, Martel N, Extremera BG, Suliman N, Campo C, et al; GESTHA Study Group.Should we perform an echocardiogram in hypertensive patients classified as having low and medium risk? Int J Cardiol. 2006;106(1):41-6. 